\title{
Modulation of amblyopia therapy following early surgery for unilateral congenital cataracts
}

\author{
I C Lloyd, J G F Dowler, A Kriss, L Speedwell, D A Thompson, I Russell-Eggitt, \\ D Taylor
}

\begin{abstract}
Background-Stimulus deprivation amblyopia is the principal cause of visual impairment in infants with unilateral congenital cataract. Even if lensectomy is undertaken at an early age, intensive postoperative occlusion of the phakic eye is essential for the development of useful vision in the aphakic eye. Despite this, the optimum method of regulating occlusion therapy is uncertain.

Methods-Interocular acuity differences identified using clinical preferential looking techniques (Keeler cards) were used to regulate target levels of phakic eye occlusion in a prospective evaluation of 10 systemically, metabolically, and neurologically normal infants in whom dense unilateral cataract was diagnosed before 8 weeks of age, and operated upon by 10 weeks. Actual occlusion levels were recorded each day by parents in a diary. The development of preferential looking acuity in the phakic and aphakic eye were compared with prediction intervals derived from observations on 43 normal children.
\end{abstract}

Results-Aphakic eye preferential looking acuities were within the normal range at last review in all but one infant. Interocular acuity differences were $\leqslant 0.5$ octave in all children older than 1 year of age at last review, and $\geqslant 1$ octave in three of four children less than 1 year old at last review (Fisher exact $p=0.033$ ). Phakic eye acuities were within the normal range in all infants at all visits.

Conclusion-Within the first 2 years of life, normal preferential looking acuity may be achieved in both eyes of infants undergoing early surgery for unilateral congenital cataract if occlusion therapy is modulated according to interocular acuity differences quantified by clinical preferential looking techniques.

(Brf Ophthalmol 1995; 79: 802-806)

Although with early surgery, accurate optical correction, and intensive occlusion a good visual outcome has become possible in infants with unilateral congenital cataract, stimulus deprivation amblyopia frequently occurs and is the major cause of visual impairment in this patient group. ${ }^{1-17}$ Occlusion of the phakic eye for a fixed period per day, ${ }^{15} 16$ adjusted according to age, may permit the development of good visual acuity in the aphakic eye, but allows no evaluation of the response to occlusion therapy, and carries risks of impaired binocularity and diplopia. ${ }^{16}$ Likewise, the use of fixation preference ${ }^{13}$ to determine the level of occlusion gives only qualitative information as to the severity of amblyopia, and is insensitive to all but gross impairment of phakic eye acuity. Quantitative measures of monocular acuity, such as those derived from pattern reversal visual evoked potentials, ${ }^{7}$ permit the detection of small reductions in phakic eye acuity, and also the calculation of interocular acuity differences which can be used to regulate the level of occlusion. Such methods may permit an excellent visual outcome but are relatively time consuming and require complex and expensive equipment. Clinical preferential looking (PL) techniques permit measures of visual acuity which correlate well with the results of laboratory based methods, ${ }^{18-20}$ but may be more rapidly, simply, and frequently obtained. ${ }^{19}$ In addition, clinical PL techniques are more sensitive in detecting deprivational than strabismic amblyopia, ${ }^{21}$ and are thus particularly suited to the detection of interocular acuity differences in monocular infantile aphakia. ${ }^{22}$ Encouraging results have been reported from pilot studies ${ }^{11} 14$ using clinical PL techniques as a guide to amblyopia therapy in infants with unilateral congenital cataract. However, no prospective evaluation of amblyopia therapy has yet been published in which interocular acuity differences (IOD) derived from clinical PL measurements has been used to regulate occlusion therapy according to a defined protocol. The current trial was designed to address this deficiency.

\section{Patients and methods}

Twelve infants with unilateral cataract diagnosed before 2 months of age, who were referred to the Hospital for Sick Children, Great Ormond Street during a 2 year period were prospectively evaluated. None showed any neurological, systemic, or metabolic abnormality. Two infants were withdrawn from the study because following lensectomy the development of retinal detachment (one) and glaucoma with corneal oedema (one) necessitated deviation from the occlusion protocol. In the remainder, gestational age ranged from 39-42 weeks (median 40 weeks), birth weight from $2.7-3.6 \mathrm{~kg}$ (median $3.5 \mathrm{~kg}$ ). Cataract was diagnosed at ages between 0 and 7 weeks, median 2 weeks and was in all cases dense and occluded the pupil. Four eyes had evidence of persistent hyperplastic primary vitreous without significant posterior involvement. Three eyes had a corneal diameter $1 \mathrm{~mm}$ 
Table 1 Motility and clinical preferential looking acuity findings

\begin{tabular}{|c|c|c|c|c|c|c|c|c|c|}
\hline Case & $P H P V$ & $\begin{array}{l}\text { Age at } \\
\text { surgery } \\
\text { (weeks) }\end{array}$ & Nystagmus & Strabismus & $\begin{array}{l}\text { Age last } \\
\text { review } \\
\text { (weeks) }\end{array}$ & $\begin{array}{l}\text { Phakic } \\
\text { eye } \\
\text { acuity }\end{array}$ & $\begin{array}{l}\text { Aphakic } \\
\text { eye } \\
\text { acuity }\end{array}$ & $I O D$ & Compliance \\
\hline $\begin{array}{r}1 \\
2 \\
3 \\
4 \\
5 \\
6 \\
7 \\
8 \\
9 \\
10\end{array}$ & $\begin{array}{l}\text { No } \\
\text { No } \\
\text { Yes } \\
\text { No } \\
\text { Yes } \\
\text { No } \\
\text { Yes } \\
\text { No } \\
\text { Yes } \\
\text { No }\end{array}$ & $\begin{array}{r}4 \\
9 \\
5 \\
10 \\
5 \\
10 \\
2 \\
7 \\
6 \\
2\end{array}$ & $\begin{array}{l}\text { None } \\
\text { None } \\
\text { None } \\
\text { Latent } \\
\text { None } \\
\text { Manifest latent } \\
\text { None } \\
\text { None } \\
\text { None } \\
\text { None }\end{array}$ & $\begin{array}{l}\text { Int XT } \\
\text { None } \\
\text { ET/Alt ET } \\
\text { Int ET/XT } \\
\text { Int XT/ET } \\
\text { ET/Alt ET } \\
\text { ET } \\
\text { None } \\
\text { Int ET/ET } \\
\text { None }\end{array}$ & $\begin{array}{r}196 \\
192 \\
124 \\
97 \\
68 \\
67 \\
44 \\
37 \\
34 \\
23\end{array}$ & $\begin{array}{l}\text { Normal } \\
\text { Normal } \\
\text { Normal } \\
\text { Normal } \\
\text { Normal } \\
\text { Normal } \\
\text { Normal } \\
\text { Normal } \\
\text { Normal } \\
\text { Normal }\end{array}$ & $\begin{array}{l}\text { Normal } \\
\text { Normal } \\
\text { Normal } \\
\text { Normal } \\
\text { Normal } \\
\text { Normal } \\
\text { Normal } \\
\text { Subnormal } \\
\text { Normal } \\
\text { Normal }\end{array}$ & $\begin{array}{l}0.5 \text { oct } \\
0.5 \text { oct } \\
0.5 \text { oct } \\
\text { None } \\
\text { None } \\
\text { None } \\
\text { None } \\
2.5 \text { oct } \\
1.5 \text { oct } \\
1.5 \text { oct }\end{array}$ & $\begin{array}{l}94 \%^{\star} \\
96 \%^{\star} \\
98 \%^{\star} \\
91 \% \\
88 \% \\
99 \% \\
95 \% \\
35 \% \\
79 \% \\
96 \%\end{array}$ \\
\hline
\end{tabular}

PHPV= persistent hyperplastic primary vitreous; $I O D=$ interocular acuity difference in octaves (oct); ET=esotropia;

$\mathrm{XT}=$ exotropia; Alt $=$ alternating; Int $=$ intermittent; Compliance $=$ ratio of actual to target occlusion level, ${ }^{\star}=$ incomplete data.

or more smaller than the fellow eye, suggestive of borderline microphthalmos (Table 1).

Lensectomy and anterior vitrectomy were performed through a limbal approach in all cases using a mechanised suction cutting instrument and anterior infusion with clearance of all lens matter except a peripheral capsular annulus. Age at surgery ranged from 2-10 weeks (median 5.5 weeks); five infants underwent surgery before 6 weeks of age. Additional surgical procedures were required in three infants and included strabismus surgery (two) and anterior segment revision (one).

Optical correction, initially with high water content contact lenses, and later with low water content or silicone rubber contact lenses, was achieved within a median of 5 days following lensectomy (range 2-19 days). A 2-3 dioptre overcorrection was prescribed; lens powers varied between 18-38 dioptres, median 30 dioptres. Although three infants required the temporary use of aphakic spectacles following squint surgery (two) and conjunctivitis (one), contact lenses were successfully used as the principal means of optical correction in all infants.

Following optical correction, clinical PL grating acuity was estimated from the infants' fixation behaviour when presented with successive pairs of targets, one target being of homogeneous grey, and the other being a vertically mounted square wave grating of spatial frequency specific to each target. Targets were circular, surrounded by a $2 \mathrm{~mm}$ white border to avoid edge artefact, ${ }^{23}$ and were presented against a homogeneous grey background at a defined distance from the infant $(38 \mathrm{~cm}$ if age $<1$ year, $58 \mathrm{~cm}$ if age $>1$ year). Testing was carried out during clinical examination, accorded with accepted presentation strategies, ${ }^{24}$ did not employ operant reinforcement, and was carried out by a single observer masked to the side of the presented grating. The clinical PL acuity findings of this observer in 10 normal infants were compared with those of another experienced observer in a masked

Table 2 The protocol determining the relation between interocular acuity difference quantified by preferential looking, and the level of occlusion prescribed until next review

\begin{tabular}{lc}
\hline $\begin{array}{l}\text { Interocular acuity difference } \\
\text { (octaves) }\end{array}$ & $\begin{array}{l}\text { Phakic eye occlusion } \\
\text { (\% waking hours) }\end{array}$ \\
\hline $0.5>$ & 50 \\
$1-1.5$ & 75 \\
$\geqslant 2$ & 100 \\
\hline
\end{tabular}

fashion according to a protocol devised by Dobson et al ${ }^{25}$; interobserver agreement was found to be excellent, all interobserver differences being $\leqslant 1$ octave (doubling of spatial frequency), and $90 \% \leqslant 0.5$ octave.

Following lensectomy, full time occlusion of the phakic eye was instituted for 1 week. After this, target levels of occlusion of the phakic eye were regulated according to a defined regimen based on the interocular PL acuity difference (Table 2). Infants were reviewed monthly, or every 14 days if undergoing full time occlusion. Actual occlusion levels, expressed as hours occluded/hours awake, were recorded each day by parents in a diary, and, from these, an average actual occlusion level was calculated for each review interval. Percentage compliance, calculated by dividing the average actual occlusion level by the target occlusion level, proved to be $\geqslant 79 \%$ in all but case no $8(35 \%)$.

Six infants were older than 1 year at most recent review (ages range 23-196 weeks, median 67 weeks); this represented approximately $90 \%$ of postnatal age. Infants were reviewed a median of 12 times in the first year.

Clinical PL acuity measurements were obtained from a control group of 43 children aged between 10 and 150 weeks, in whom no ocular abnormality, refractive error, or strabismus was identified. Regression models were formulated for right and left eyes based on log transformations of PL acuity and age. These showed good fit $\left(R^{2}=0.74 \quad p=0.0005\right.$, $R^{2}=0.70 p=0.0005$ respectively), and no difference between eyes (paired $t$ test log PL acuity right eye $=\log \mathrm{PL}$ acuity left eye, $\mathrm{p}=0.65$ ). By calculating the standard error of the forecast, $95 \%$ prediction intervals of $\mathrm{PL}$ acuity versus age were constructed for each eye, and these were used to provide a normal range (Fig 1) with which to compare acuities in infants with cataract. Fisher's calculation of exact probability was used to compare the proportion of interocular acuity differences $\geqslant 1$ octave before and after 1 year of age.

Informed consent was obtained from guardians or parents of all children participating in the study. Ethical approval was obtained from the ethics committee of the Hospital for Sick Children.

\section{Results}

A tendency towards normalisation of aphakic eye acuity by 1 year of age was apparent 


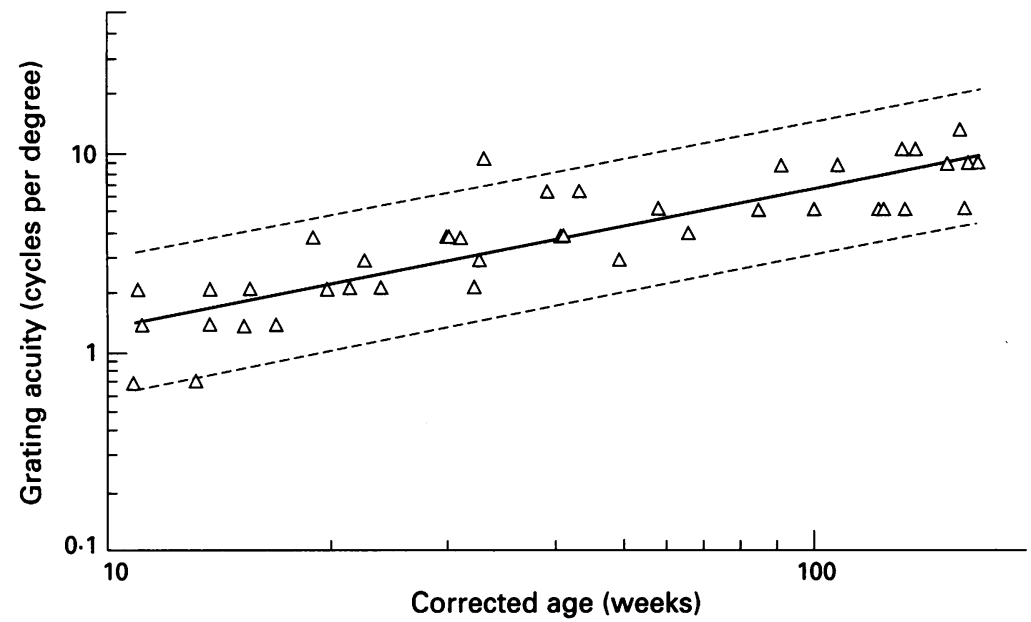

Figure 1 Relation between preferential looking acuity and age in the left eyes of 43 normal children aged between 10 and 150 weeks, together with 95\% prediction intervals (normal range).

(Fig 2). Aphakic eye acuity was within the normal range at last review in all but one child with poor compliance (35\%) (Fig 3).

Clinical PL acuities in the phakic eyes of all 10 infants were within the normal range at last review and all preceding visits. However, on one occasion in each of cases 3-6, a drop in phakic eye acuity of $0 \cdot 5-1$ octave was noted between successive visits, 1-6 weeks apart, in association with comparable rises in aphakic eye acuity (0.5-1.5 octaves) (for example, Fig 4). These episodes occurred at ages of 11-49 weeks, following periods of phakic eye occlusion ranging from $72-94 \%$ of waking hours. Phakic eye acuity invariably recovered when occlusion was reduced to $50 \%$ in accordance with the protocol.

Interocular acuity differences were half an octave or less at last review in all infants followed beyond the age of 1 year, whereas in three of four infants less than 1 year of age at last review the IOD was $1-2.5$ octaves (Fisher exact, $p=0.033$ ) (Fig 3 ). The largest IOD (2.5 octaves) was recorded in the infant with the poorest compliance $(35 \%$, case 8$)$.

\section{Discussion}

In this study of monocular infantile aphakia, we report normal clinical PL acuity in the

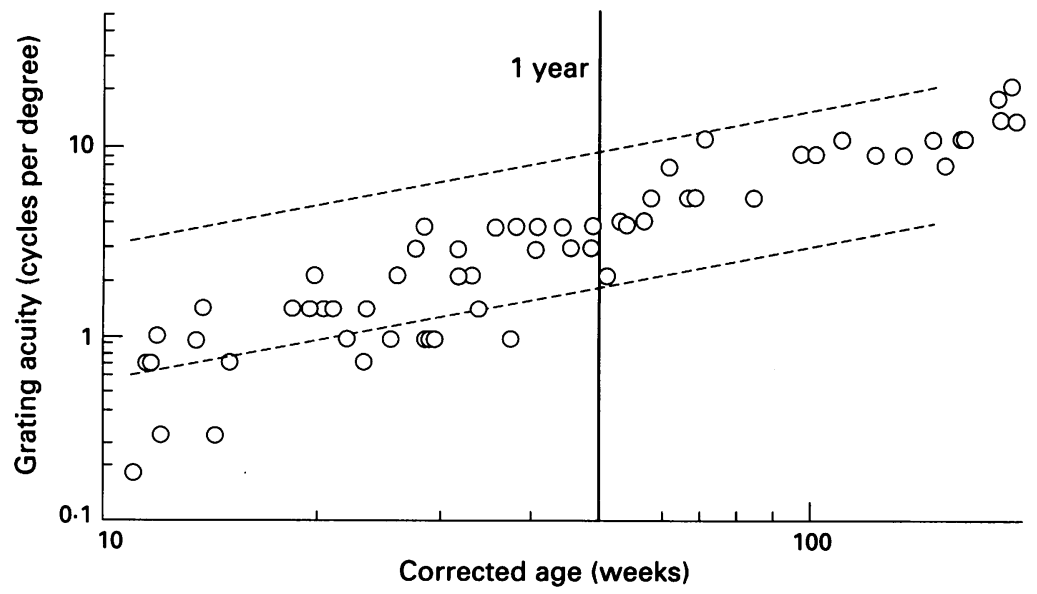

Figure 2 Aphakic eye preferential looking acuities - all measurements from all 10 infants - as a function of age, with normal ranges (broken lines). aphakic eye of nine of 10 infants. A variety of factors may explain this finding. Surgery was carried out before 10 weeks of age in all infants, and before 6 weeks in five infants. Thus according to the definitions of Birch, ${ }^{17}$ half had 'early surgery', and half 'very early surgery', with the attendant prospect of good aphakic eye acuity. Lensectomy, with removal of the posterior lens capsule and anterior vitrectomy, was employed in all cases, thereby eliminating the risk of amblyopia from posterior capsular opacification. Optical correction using contact lenses was undertaken shortly after surgery, was well tolerated and frequently checked. The absence of coexistent neurological or systemic dysfunction prevented any adverse effect such abnormalities may have on visual development. The good visual results obtained cannot, however, be attributed to incomplete obstruction of the visual axis due to partial or lamellar cataract, because all infants had dense opacities completely obstructing the pupil. In addition, because cataract was diagnosed on average 2 weeks after birth, and no later than 7 weeks, the achievement of good acuities cannot be explained by assuming that the cataracts were developmental in nature. It is notable also that the presence of borderline microphthalmos, as shown by a $1 \mathrm{~mm}$ disparity in horizontal corneal diameter between eyes (three eyes), and minor degrees of persistent hyperplastic primary vitreous (four eyes) did not prevent the achievement of satisfactory acuity levels.

The frequent use of clinical PL acuity measures provided a sensitive method of identifying and quantifying amblyopia, a means of titrating the intensity of occlusion therapy to the severity of amblyopia, and an index of the response to therapy. In addition, it permitted parents to appreciate the functional results of their efforts at occlusion, thereby enhancing compliance. The use of a diary in which occlusion levels were recorded on a daily basis also provided a valuable took in quantifying compliance and adjusting occlusion therapy. Parents frequently used the diary to evaluate their own compliance, and modified occlusion levels to approximately more closely to the target $-\mathbf{a}$ form of homeostasis. The generally high levels of compliance $(\geqslant 79 \%)$ which resulted seem likely to be a major factor in determining eventual visual performance, and it is notable that the infant (case 8) with subnormal aphakic eye acuity and the largest interocular acuity difference $(2.5$ octaves) showed substantially the lowest compliance (35\%).

Phakic eye acuities remained within the normal range in all infants throughout the study. In four infants, however, episodes were noted in which a fall in phakic eye acuity occurred in association with a comparable rise in aphakic eye acuity over a short time period during which occlusion was intense. Phakic eye acuity invariably recovered when occlusion was reduced. Intensive occlusion may likewise produce a reduction in the amplitude of pattern visual evoked potentials in the phakic eye of infants with monocular 


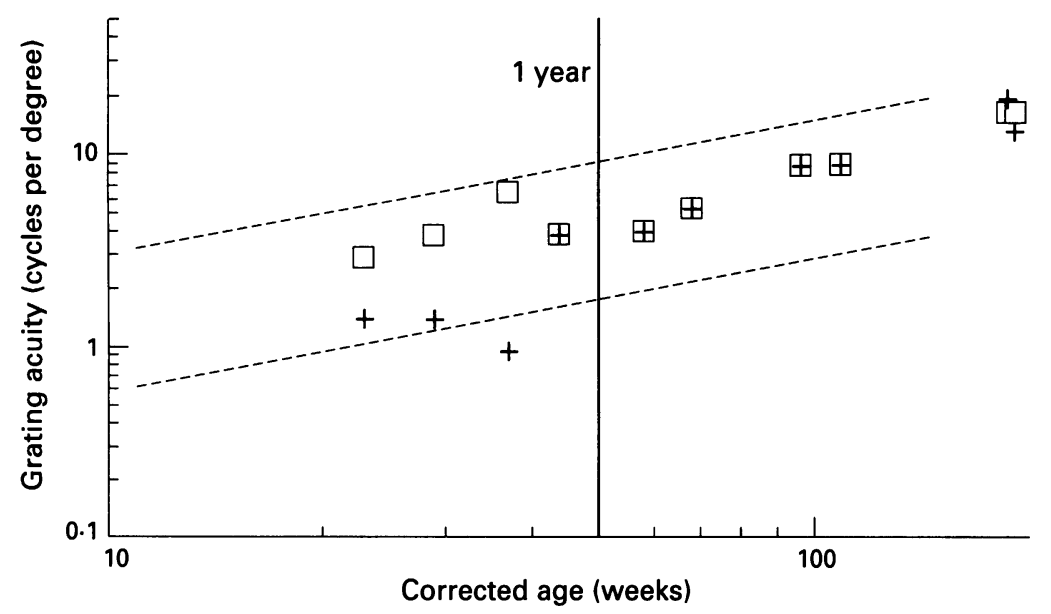

Figure 3 Phakic eye ( $\square$ ) and aphakic eye (+) clinical preferential looking acuities at last review, with normal ranges (broken lines).

congenital cataract, even in the absence of linear acuity deficit. ${ }^{26}$ It is not known, however, whether prolonged periods of high level occlusion have an adverse effect on eventual visual performance in the phakic eye. Although abnormalities of contrast sensitivity at high spatial frequencies and subtle linear acuity deficits have been reported in phakic eyes of children with monocular congenital cataract, ${ }^{27}$ the levels of occlusion used in this study varied considerably, and the cause of the deficits is unclear. A later study demonstrated no phakic eye deficits in contrast sensitivity or linear acuity, and questioned the validity of the earlier findings. ${ }^{17}$ It does appear likely, however, that intensive occlusion may adversely affect long term prospects of binocularity. Although impaired binocularity has been regarded as typical in infants with monocular cataract, the incidence of strabismus being of the order of $70 \%,{ }^{17}$ it is now recognised that some children may develop useful binocularity with stereoacuities of 50-310 seconds of arc. ${ }^{17} 28-30$ By contrast, if occlusion is intense and long term, severely impaired binocularity and diplopia may result. ${ }^{16}$ Clinical PL acuity measures, by identifying any drop off in phakic eye acuity, may signal the need to reduce occlusion and thereby lessen any potential threat to binocular development.

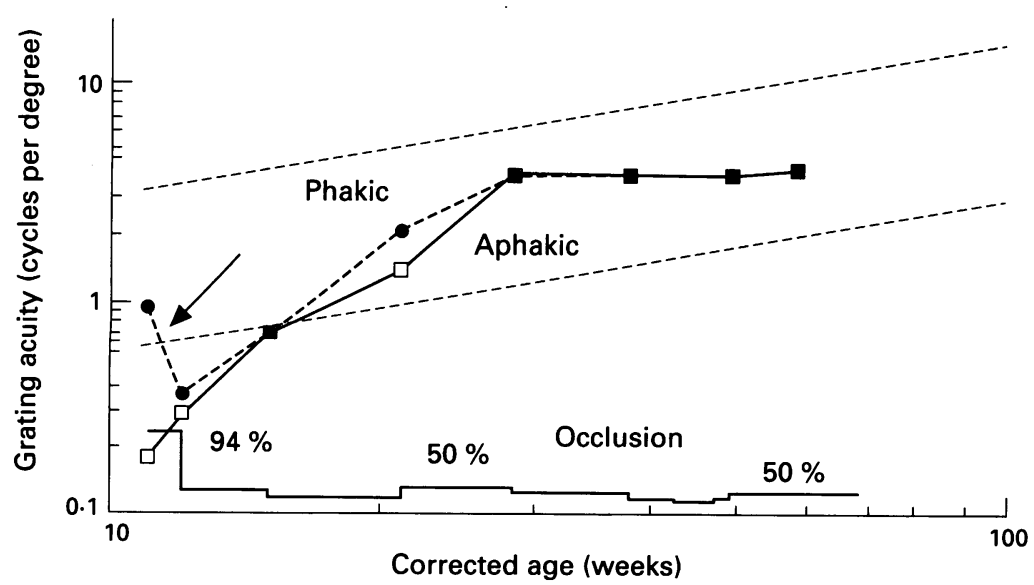

Figure 4 Visual development plot of case 6. Aphakic (+) and phakic ( $\square$ ) eye clinical preferential looking acuities, normal ranges (broken lines), and occlusion levels. Arrow indicates reduction in phakic eye acuity with intensive occlusion.
In this study visual development was plotted by comparing clinical PL acuity measurements in infants with cataract with $95 \%$ prediction intervals derived from observations on 43 normal children. Although the fit of the regression model applied to the normative data was in general good $\left(R^{2}=0 \cdot 70, p=0.00005\right)$, the variance of $\log$ PL acuity decreased slightly with increasing age. Results were, however, comparable with those of other workers. ${ }^{20} 3132$ Other potential sources of error exist in this approach to the assessment of visual development. PL acuity, although correlated with recognition acuity, appears to overestimate it by approximately 1 octave or 2 Snellen lines. ${ }^{22}$ In addition, PL acuity methods are relatively less sensitive to strabismic than to deprivational amblyopia, ${ }^{21}$ and given the high incidence of strabismus $(70 \%)$ some visual impairment may conceivably have remained undetected. However, the development of strabismic amblyopia is unlikely given the levels of phakic eye occlusion, and therefore any such effect is unlikely to be significant. By contrast, the use of vertically mounted gratings, as in this study, may have resulted in an underestimation of aphakic eye acuity in the two infants with latent nystagmus. ${ }^{33}$ Finally, there is evidence that PL acuity may plateau at ages above 24 months ${ }^{122}$ in intensively occluded monocularly aphakic infants, a phenomenon described as asymptotic visual development. However, this evidence is derived from studies in which occlusion therapy was not based on PL acuity findings and it is conceivable that with the application of a protocol such as ours, directed at the eradication of interocular acuity differences, such a process may not occur. There is also some indication that in infants undergoing very early surgery, as in five cases in this study, asymptotic visual development occurs later and is less marked. ${ }^{17}$

The use of clinical PL techniques to quantify interocular acuity differences allows titration of the level of occlusion to the severity of amblyopia, and offers the prospect of normal grating acuity in both eyes of infants with monocular congenital cataract. In addition, by identifying reductions in phakic eye acuity, any potential risk to the phakic eye or the development of binocularity which may result from intensive occlusion may be minimised. We routinely employ clinical PL techniques to regulate the management of amblyopia in infants with monocular congenital cataract.

Mr Lloyd's research fellowship was supported by the Ulverscroft Foundation. Our thanks to Christine Timms and Lucy Stimson for their work in the congenital cataract clinic, to the staff of the Great Ormond Street creche, and the parents of the staff of the Great Ormond Street creche, and the parents of
children attending the creche. Thanks also to Drs A J Moghissi children attending the creche. Thanks also to Drs A J Moghissi
and R J Halvorsen, to the staff of the Holborn Health Centre and R J Halvorsen, to the staff of the Holborn Health Centre
cell Baby Clinic, and the parents of children attending the
clinic. linic. The authors have no proprietary interest in Keeler acuity
cards.

1 Frey T, Friendly D, Wyatt D. Reevaluation of monocular cataracts in children. Am $\mathcal{f}$ Ophthalmol 1973; 76:
381-8.

2 Hiles DA, Waller PH. visual results following infantile cataract surgery. Int Ophthalmol Clin 1977; 17: 265-82.

3 Hiles DA. Visual acuities of monocular IOL and non-IOL aphakic children. Ophthalmology 1980; 87: 1296-300. 
4 Pratt-Johnson JA, Tillson G. Visual results after removal of congenital cataracts before the age of 1 year. Can $\mathcal{F}$ Ophthalmol 1981; 16: 19-21.

5 Jacobson SG, Mohindra I, Held R. Development of visual acuity in infants with congenital cataracts. $\mathrm{Br} \mathcal{F}$ Ophthalmol 1981; 65: 727-35.

6 Odom JV, Hoyt CS, Marg E. Effect of natural deprivation and unilateral eye patching on visual acuity of infants and children. Arch Ophthalmol 1981, 99: 1412-6.

7 Beller R, Hoyt CS, Marg E, Odom JV. Good visual function after neonatal surgery for congenital monocular cataract. Am $\mathcal{F}$ Ophthalmol 1981; 91: 559-65.

8 Parks MM. Visual results in aphakic children. $A m f$ Ophthalmol 191982; 94: 411-9.

9 Ben Ezra D, Paez JH. Congenital cataract and intraocular lenses. Am F Ophthalmol 1983; 96: 311-4.

10 Robb RM, Mayer DL, Moore BD. Results of early treatment of unilateral congenital cataracts. $\mathcal{f}$ Pediatr ment of unilateral congenital catara

11 Catalano RA, Simon JW, Jenkins PL, Kandel GL. Preferential looking as a guide for amblyopia therapy in monocular infantile cataracts. $f$ Pediatr Ophthalmol Strabismus 1987; 24: 56-63.

12 Birch EE, Stager DR, Wright WW. Grating acuity development after early surgery for congenital unilateral cataract. Arch Ophthalmol 1986; 104: 1783-7.

13 Drummond GT, Scott WE, Keech RV. Management of monocular congenital cataracts. Arch Ophthalmol 1989, 107: 45-51.

14 Lorenz B, Worle J. Visual results in congenital cataract with the use of contact lenses. Graefes Arch Clin Exp Ophthalmol 1991; 229: 123-32.

15 Cheng KP, Hiles DA, Biglen AW, Pettapiece MC. Visual results after early surgical treatment of unilateral congenital cataracts. Ophthalmology 1991; 98: 903-10.

16 Pratt-Johnson JA, Tillson G. Unilateral congenital cataract; binocular status after treatment. $\mathcal{f}$ Pediatr Ophthalmol Strabismus 1989; 26: 72-5.

17 Birch EE, Swanson WH, Stager DR, Woody M, Everett M. Outcome after very early treatment of dense congenital unilateral cataract. Invest Ophthalmol Vis Sci 1993; 34 3687-99.

18 Sebris SL, Dobson V, McDonald M, Teller D. Acuity cards for visual acuity assessment of infants and children in clinical settings. Clin Vis Sci 1987; 2: 45-58.

19 Preston KL, McDonald M, Sebris SL, et al. Validation of the acuity card procedure for assessment of infants with ocular disorders. Ophthalmology 1987; 94: 644-53.

20 Brown AM, Yamamoto $M$. Visual acuity in newborn and preterm infants measured with grating acuity cards. Am $f$ Ophthalmol 1986; 102: 245-53.

21 Ellis GS, Hartmann EE, Love A, May JG, Morgan KS Teller acuity cards versus clinical judgement in the diag-
nosis of amblyopia with strabismus. Ophthalmology 1988; 95: $788-90$.

22 Mayer DL, Moore B, Robb RM. Assessment of vision and amblyopia by preferential looking tests after early surgery for unilateral congenital cataracts. $\mathcal{F}$ Pediatr Ophthalmo Strabismus 1989; 26: 61-8.

23 Robinson J, Moseley MJ, Fielder AR. Grating acuity cards spurious resolution and the 'edge artifact'. Clin Vis $S c i$ 1990; 6: 85-6.

24 McDonald MA, Dobson V, Sebris SL, Baitch L, Varner D, Teller DY. The acuity card procedure: a rapid test of infant acuity. Invest Ophthalmol Vis Sci 1985; 26: infant act

25 Dobson V, Carpenter NA, Bonvalot K, Bossler J. The acuity card procedure: interobserver agreement in infants with perinatal complications. Clin Vis Sci 1990; 6: 39-48.

26 Lloyd IC, Goss-Sampson M, Jeffrey BG, Kriss A, RussellEggitt I, Taylor D. Neonatal cataract: aetiology, pathogenesis, and management. Eye 1992; 6: 184-96.

27 Lewis TL, Maurer D, Tytla ME, Bowering ER, Brent HP Vision in the 'good' eye of children treated for unilatera congenital cataract. Ophthalmology 1992; 99: 1013-7.

28 Gregg FM, Parks MM. Stereopsis after congenital monocular cataract extraction. Am $\mathcal{f}$ Ophthalmol 1992; 114: 314-7.

29 Tytla ME, Lewis TL, Maurer D, Brent HP. Stereopsis after congenital cataract. Invest Ophthalmol Vis Sci 1993; 34: 1767-73.

30 Wright KW, Matsumoto E, Edelman PM. Binocular fusion and stereopsis associated with early surgery for monocula congenital cataract. Arch Ophthalmol 1992; 110: 1607-9.

31 Chandna A. Natural history of the development of visua acuity in infants. Eye 1991; 5: 20-6.

32 Katz B, Sireteanu R. Teller acuity test card: a useful method for the clinical routine? Clin Vis Sci 1990; 5: 307-23.

33 Meiusi RS, Lavoie JD, Summers CG. The effect of grating orientation on resolution acuity in patients with nystagmus. $\mathcal{f}$ Pediatr Ophthalmol Strabismus 1993; 30: 259-61. 